\title{
Guidance for the planning and design of genitourinary medicine clinics
}

\author{
R N T Thin, Jane Lamb
}

\begin{abstract}
The number of patients attending genitourinary medicine (GUM) clinics in Britain has risen greatly in recent years. In contrast there have been very modest increases in staff and there are no data on accommodation or facilities. The Department of Health therefore set up a team to provide guidance on the planning and design of GUM clinics. After a survey the team concluded that GUM clinics in England were poorly and inappropriately accommodated. In their guidance they recommended that GUM clinics are sited in general outpatient departments (OPDs) of general hospitals; this improves patient care simplifying cross referral, consultation and other aspects of operational efficiency and convenience for patients, improves cost effectiveness and increases convenience for staff. Some facilities may be shared. Reception should be an attractive focal point and allow patients to be received and registered in privacy. Facilities for clinical examination are the central part of a clinic. Rooms which allow consultation and examination (CE) rooms of male and female patients maximise efficiency, flexibility and confidentiality. Sound attenuation is vital and decor should be attractive so inspiring confidence by producing a noninstitutional atmosphere. An adequate number of CE rooms is essential and the waiting area should be sufficient for booked and walk in patients plus companions. There must be rooms for venepuncture, treatment and colposcopy. Interviewing rooms and offices are important, and there must be a secure health records store. A childrens play area and staff accommodation can be shared. An area for handling and simple investigation of patients' specimens is essential. This guidance should be interpreted flexibly. Though commissioned by
\end{abstract}

Department of Genitourinary Medicine, St Thomas' Hospital, London, UK

R N T Thin

Estates Directorate 6, Department of Health, Euston

Towers, London, UK

Jane Lamb the Department of Health for England, it is hoped that it will be useful for planning and designing genitourinary medicine clinics throughout Britain and in other countries.

\section{Introduction}

Since World War 2 there has been a massive increase in the number of patients presenting to genitourinary medicine (GUM) clinics in England. ${ }^{2}$ In contrast there have only been modest increases in staff, ${ }^{1}$ and there are no data on facilities. In 1988 the Department of Health (D of $\mathrm{H}$ ) undertook a survey of GUM clinics and published a report frequently called the Monks Report. ${ }^{3}$ The survey found the general standard of accommodation for GUM clinics was poor. The report recommended that the standard of accommodation for GUM clinics should be no less than that for general outpatient departments (OPDs), and recommended that the Special Treatment Clinic Design Guide 1974, ${ }^{4}$ be revised. In 1989 the $\mathrm{D}$ of $\mathrm{H}$ set up a team to prepare a supplement on GUM clinics to their guidance on building OPDs. ${ }^{5}$ The core team of four professionals comprised an architect from the $\mathrm{D}$ of $\mathrm{H}$ Estates Directorate, an administrator, a GUM consultant, and a GUM clinic nurse, who were supplemented by an engineer and quantity surveyor. The guidance was published at the end of $1989 .^{6}$ This paper summarises the recommendations and provides some background information.

\section{Method}

The core team visited eight clinics selected to represent the range of clinics in England; these included large full time clinics in major cities and part time clinics in small towns. Some clinics were in modern purpose built premises and others in old dilapidated adapted buildings. The team examined the premises, obtained data on work load and consultation times, and studied patient flow and working practices. Staff were asked to time the duration of consultations. Format, storage and retrieval of health records were also studied. In addition the whole team visited three clinics, a new one in large purpose built premises, a part time clinic in a modern general $O P D$, and the third in small dedicated old adapted premises. Standard questionnaires were completed 
for all clinics during the visit.

By means of a simpler questionnaire the team obtained data on accommodation, workload, and consultation times from an additional four clinics. Data were obtained from a further two clinics on storage and retrieval of health records, and from the $\mathrm{D}$ of $\mathrm{H}$ on total attendances (patient visits) and number of sessions per annum in all GUM clinics in England.

\section{Findings}

Data for England supplied by the Department of Health Data supplied by the D of $\mathrm{H}$ showed that of the 199 Health Districts in England in 1987/88, 47 had no GUM clinic, 68 reported total attendances of 5000 or less, 42 reported attendances of 5001 to 10,000 , and the remainder reported attendances over 10,001 (table 1).

Reported total attendances at GUM clinics during $1987 / 88$ in England were $1.5 \times 10^{6}$. Total number of clinic sessions were $5.69 \times 10^{4}$, giving 34.3 attendances per session; duration of sessions is unclear but they are usually 3 to 3.5 hours which in practice may actually continue for 4 hours. These data give $5 \cdot 2$ minutes per attendance for a 3 hour session, $6 \cdot 1$ minutes for 3.5 hour session or $\mathbf{7 . 0}$ minutes for a 4 hour session.

\section{Data on clincs surveyed}

Total attendances for $1987 / 88$ at clinics visited ranged from 3000 to approximately 68,000 and at those assessed by questionnaire from 4400 to 7260 . Sessions worked per annum varied from 200 to 520 giving attendances per session varying from 22 to 69 with a mean of 34.5 and a median of 29 .

Of the eight clinics visited by the core team, four were in separate buildings and four were integral with a general OPD. Three had separate entrances for male and female patients, with separate access to reception and waiting areas. The remainder had a single entrance, common access to reception and a common initial waiting area. The patients and staff in clinics that were integral and with single entrances and waiting areas liked the arrangement. This included large city clinics and small clinics in small

Table 1 Attendance at GUM clinics by health district $1987 / 88$

\begin{tabular}{ll}
\hline Number of attendances & Number of districts \\
\hline None & 47 \\
$1-5,000$ & 68 \\
$5,001-10,000$ & 42 \\
$10,001-20,000$ & 24 \\
$20,001-30,000$ & 8 \\
$30,001-40,000$ & 6 \\
40,001 and over & 4 \\
\hline
\end{tabular}

towns. Patients and staff in isolated clinics were also satisfied. Only one clinic completing the questionnaire gave an answer on entrance and waiting arrangements.

The numbers of chairs in the waiting areas ranged from 10 to 55 with a mean of 22 and a median of 15 . The number of annual attendances per chair varied from 150 to 882 with a mean of 532 and a median of 515.

Clinics varied in their arrangements of separate consulting and examination rooms, and combined rooms for both purposes. The total number of these rooms available for routine GUM cases, as compared with specialist purposes such as colposcopy, ranged from one to nine. The total attendances per room were 750 to 7555 with a mean of 2774 per year and a median of 1875 .

Consultation times varied from 32 minutes to see a new female at her first attendance to 5 minutes to see a male on a return visit (table 2 ) with a mean of 15.4 minutes.

Six of the 12 clinics provided separate accommodation for treatment, only four had separate accommodation for venepuncture and in one clinic venepuncture and treatment were undertaken in the same space. In the remainder venepunctures were undertaken in the examination rooms. Accommodation for colposcopy and dedicated space for patients with human immunodeficiency virus (HIV) infection varied according to the size of the clinic and the demand.

The format of health records differed with some clinics using standard hospital folders and stationery, and others using special records. The number of health records stored per foot of shelving varied from 40 to 125 . Approximately 200 new records were created for each 1000 total attendances per year. After storage for four years without use only seven records were retrieved per 1000 total attendances per year.

Table 2 Consultation times in minutes

\begin{tabular}{llll} 
& & Males & Females \\
\hline Clinics Visited & & \\
First visit & maximum & 30 & 32 \\
& minimum & 10 & 20 \\
& mean & $21 \cdot 5$ & $25 \cdot 5$ \\
Return visit & maximun & 10 & 15 \\
& minimum & 5 & 10 \\
& mean & 8 & 13 \\
Clinics Questioned & & \\
First visit & maximum & 20 & 20 \\
& minimum & 10 & 15 \\
Return visit & maximun & 14 & $15 \cdot 3$ \\
& minimum & 10 & 15 \\
Overall mean & 8 & 9 \\
mean & $9 \cdot 3$ & $13 \cdot 3$ \\
& & &
\end{tabular}


Facilities for handling patients' specimens varied from dedicated first class accommodation to cramped shared space. A dedicated interviewing room for health advisers in sexually transmitted diseases (HAs) was available in six of the eight clinics visited but in only one of the four clinics questioned. Consultants had offices in seven of the 12 clinics, secretaries had their own office in eight clinics but two were of a poor standard, and sisters had offices in seven clinics but three were poor-cramped, containing extra furniture and stores and in poor decorative order. Seven clinics had staff rest rooms but these were cramped and in poor decorative order in two. Accommodation for interviewing couples or difficult time consuming cases was generally inadequate.

In many clinics the design prevented smooth patient flow despite efforts by staff to maximise the use of their accommodation. Storage space was frequently inadequate so stores obstructed corridors adding to the confusion.

\section{Recommendations for building a GUM clinic Siting}

The notes published with the Public Health (Venereal Diseases) Regulations of 1916 recommended that clinics be organised in suitable general hospitals. ${ }^{8}$ The Monks Report recommended that GUM clinics be located in general OPDs of district general hospitals. ${ }^{3}$

The guidance for the planning and design of general OPDs ${ }^{5}$ recommends siting near the main hospital entrance, with access via this entrance though sometimes an OPD may have its own entrance. The entrance should be clearly sign posted and the appearance welcoming. General OPDs are divided into suites in which a number of clinic rooms can be operated singly or together with an associated reception and waiting area. ${ }^{5}$ All this suits GUM, provided certain facilities are added. As with other clinics, GUM within a general OPD can share facilities such as venepuncture. Up to $50 \%$ additional spaces may be required for a self contained GUM clinic. ${ }^{6}$ Practicing GUM in a general OPD improves patient care, simplifying cross referral, consultation and other aspects of operational efficiency and convenience for patients, improves cost effectiveness, and increases convenience for staff. ${ }^{5}$ GUM clinics should be sited within easy reach of outpatient clinics such as gynaecology and dermatology, and conveniently located for supporting departments such as microbiology, virology and radiology. ${ }^{6}$

\section{Accommodation and patient flow}

The recommended accommodation is summarised in the figure. The environment should be aesthetically attractive and inspire confidence in the service

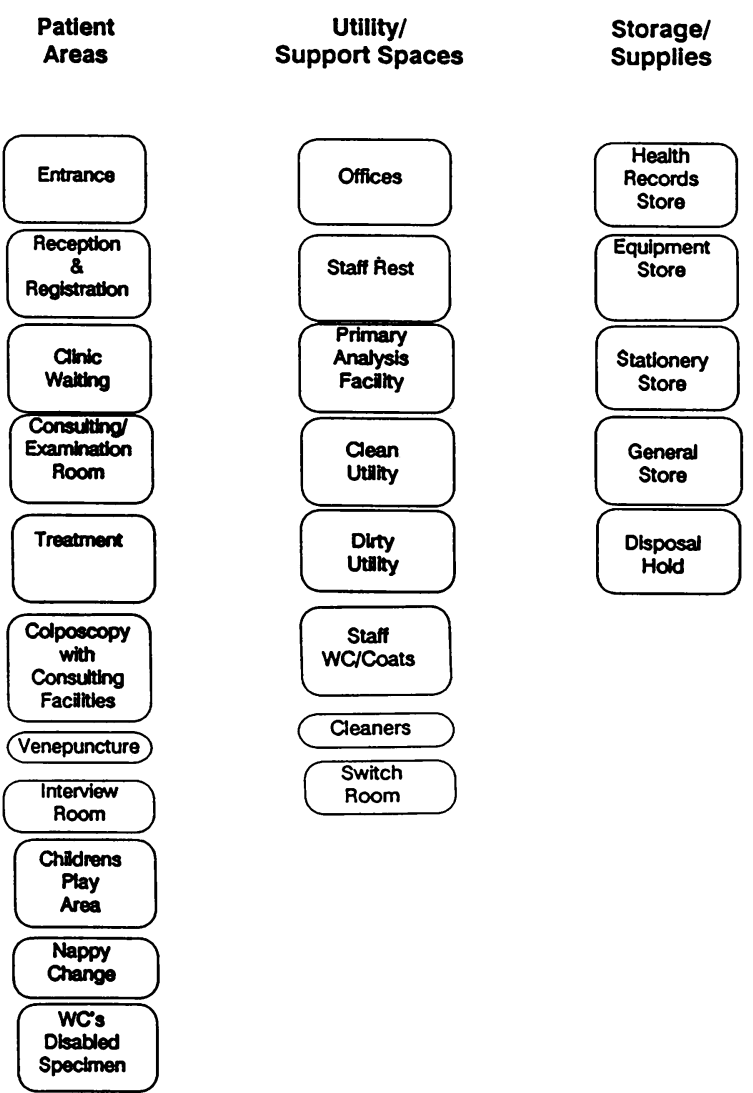

Figure Accommodation for $G U$ medicine clinic.

offered. The spaces should be arranged to allow a smooth flow of patients attending for consultation with a doctor. Arrangements should allow patients to attend by appointment or to walk in and be seen without an appointment. After their initial consultation, patients may be asked to attend only for treatment and arrangements should allow a smooth flow for these visits.

Sizing

The data in table 1 suggested that it would be convenient to consider three sizes of clinic; those with total attendances up to 5000 a year (small clinic), 5001 to 10,000 a year (medium clinic), and over 10,000 a year (large clinic). Guidance for accommodation is given in table 3 . The number of $\mathrm{CE}$ rooms is based on a consultation time of 18 minutes, and a total number of attendances of 15,000 for the large clinic. The numbers in table 3 are examples; local planners should adjust these according to their needs.

Access and waiting area

A single entrance and waiting area is recommended for male and female patients. This will allow couples 
Table 3 Guidance for accommodation in 3 sizes of clinics

\begin{tabular}{lccc}
\hline $\begin{array}{l}\text { Size } \\
\text { Attendances per year }\end{array}$ & $\begin{array}{c}\text { Up to } \\
5000\end{array}$ & $\begin{array}{c}5001 \text { to } \\
10,000\end{array}$ & $\begin{array}{l}10,001 \\
\text { and over }\end{array}$ \\
\hline CE rooms & 3 S $\dagger$ & 5 D $\ddagger$ & 7 D \\
Treatment rooms & 1 S & 1 D & 1 D +1 S \\
Colposcopy room & 1 S & 1 S & 1 D \\
HA Interviewing rooms & 1 S & 1 D & 2 D \\
Offices & 1 S & 3 D & 5 D \\
No. of chairs & 11 & 18 & 30 \\
$\quad$ general & 4 & 6 & 12 \\
\hline
\end{tabular}

*Based on 15,000 attendances per year

†Shared-but for exclusive use of GUM during clinic sessions $\ddagger$ Dedicated to GUM whole time.

to enter and wait together. The number of seats in the waiting area should be sufficient for appointment and walk in patients, and for friends and relatives accompanying them. The number of chairs in table 3 allows a mean of 521 visits per chair per year for all attendances in the general clinic falling to 374 per chair if one includes the chairs for colposcopy etc. Colour schemes, soft floor coverings, comfortable chairs, relevant up-to-date attractive reading material, soft music, and videos all assist in creating a relaxed non-institutional atmosphere which helps patients and staff. The atmosphere should be similar to that in any other OPD, emphasising the similarities between attending GUM and other clinics.

\section{Reception}

The position, lighting and decor of reception should make it an attractive focal point, and the design must enable patients to be received and registered in privacy without creating any feeling of a barrier between patient and receptionist. The design should allow staff to observe the whole waiting area giving the patient a feeling of reassurance and contact with staff. Receptionists must also have easy access to the health records store and to the clinical area.

\section{Childrens' play area}

More children accompany adults attending clinics now than in the past and whole families may attended together. It is important that an area be provided for children to play in safety and under supervision.

\section{Consulting and examination rooms}

Guidance for building general OPDs ${ }^{5}$ recommends combined consulting and examination (CE) rooms. They allow an unbroken consultation and if there is only one door, enhance accoustic privacy. The combined room may be used for consultation, examination or both. Males and females may be seen. In addition, any such room may be used for prolonged interviewing if needed, and for treatment and other procedures. A suite of such rooms provides maximum flexibility and utilisation. Each room should be large enough to accommodate a desk, a couch arran- ged to allow access to both sides and with surrounding curtains. There must be a wash hand basin and enough space for chairs and trolleys for equipment. Arrangements should permit maximum privacy especially when the door is opened. All these factors apply to GUM where it is vital to ensure privacy and confidentiality to maximise patients' confidence, so combined rooms are recommended. ${ }^{6}$ Couches should be fitted with fold away stirrups for the examination of females in the lithotomy position.

\section{Clinic laboratory or primary analysis facility}

Clinic staff have to collect samples from patients, to undertake simple investigations such as staining and microscopy, and store specimens pending their transfer to the laboratory. Today the term laboratory frequently implies the need for special facilities such as ventilation, and gas and fume exhaust. Such facilities are not required in a GUM clinic so the term Primary Analysis Facility (PAF) is suggested. ${ }^{6}$ The necessary facilities can be provided in a CE room in a part time clinic, though dedicated space is required in a full time clinic. A refrigerator, incubator and centrifuge are essential; if these cannot be kept in a $\mathrm{CE}$ room in a part time clinic, they may be located in the dirty utility room.

\section{Treatment room}

All clinics must have a treatment room where routine treatments such as drug therapy are administered or dispensed, where injections can be given, and warts treated in conditions of privacy. ${ }^{6}$ There should therefore be a desk, chairs, a couch with fold away stirrups and space for equipment. Wart treatment may generate odours but the standard of ventilation provided in treatment rooms will be sufficient to remove these. ${ }^{5}$

\section{Colposcopy room}

In smaller clinics colposcopy may be undertaken part time in a CE room. In a larger clinic a dedicated room is needed; this requires space for consulting, a couch, a colposcope and treatment modalities such as cold coagulation, loop diathermy or laser. Treatment and colposcopy sessions may be organised with separate appointment systems so a subsidiary waiting area may be needed.

\section{Interviewing rooms}

Suitable rooms for HAs for interviewing and counselling are essential, near the CE rooms. ${ }^{6}$ They must be sound attenuated, and furnished and decorated to ensure maximum patient confidence. Interviewing rooms are also needed by clinical psychologists and others.

\section{Offices}

Offices are normally only provided in general OPDs 
for a manager, sister and for visiting staff. ${ }^{5}$ GUM consultants, unlike consultants in other disciplines, are based in full time clinics, so require dedicated offices. ${ }^{6}$ Offices are also essential for a secretary, other doctors and health advisers. ${ }^{6} \mathrm{~W}$ iring is provided for computers in OPDs ${ }^{5}$; In GUM clinics space will be needed for computer terminals to link with the hospital system and in many instances for dedicated departmental computers.

\section{Health record storage}

Health record storage space will depend on the thickness of paper notes and the numbers generated. Full advantage should be taken of microfilm and microfiche; there will be advantages in miniaturising records which have not been required for about four years. The confidentiality of records must be preserved and it is essential that the storage space is securely locked when not in use and access is limited to those who require to see them.

\section{General storage}

With the advent of disposable items of equipment adequate storage space is essential.

\section{Comments}

The findings in this survey supported those of the Monks Report that GUM clinics in England were poorly and inappropriately accommodated. ${ }^{3}$ It was agreed that the standard for GUM should be not less than that for general OPDs. The decision to recommend location in general OPD's was made after studying integral and isolated clinics. Staff in clinics in general OPDs found this improved working practices and was liked by patients. Staff and patients considered that confidentiality could be preserved. Although staff working in isolated clinics claimed that the patients preferred such an arrangement, this appeared more related to historical custom than modern practice. The same arguments applied to a single entrance and waiting area. The layout of general OPDs in a modern "Nucleus Hospital" provides functional division into suites which give the impression of physical separation, preserves confidentiality, and suits GUM. This design helps patients to feel attending a GUM clinic is like attending any other OPD. Good quality care is easier to provide in an integral department and is in keeping with modern concepts of genitourinary medicine.

A crucial problem was to decide on the best simple measure of workload. The most accurate single measure to apply to accommodation appeared to be total attendances.

Size of accommodation is critical. Advice on sizes is given in guidance for general OPDs, ${ }^{5}$ but this does not include the additional requirements of GUM. Fundamental to our calculations was the duration of consultation. The figures quoted are derived from a mean of all methods used to obtain these times. The facilities for the clinical examination of patients are the central part of a clinic and we concluded that combined CE rooms are preferable. In his model clinic design of 1919 Harrison $^{9}$ recommended that the patient remained in one room for continuity of consultation. In the large clinic it might be more economic to equip some rooms for male patients, some for females and some for both, though this will reduce flexibility. The numbers of $\mathrm{CE}$ rooms recommended are based on a consultation time for general GUM visits of 18 minutes. This is closer to the 20 minutes recommended by the Royal College of Physicians, ${ }^{10}$ than the 13.4 minutes in the Monks Report, ${ }^{3}$ or the $5 \cdot 2$ to 7.0 minutes derived from the $\mathrm{D}$ of $\mathrm{H}$ data. The waiting area must provide adequate space for booked and walk in patients and companions.

All GUM clinics have their own health records store. It is anticipated that the small clinic will be part time so all the rest of their accommodation will be shared though used solely by GUM when the clinic is open. The medium and large clinics will be full time. Unlike half the clinics surveyed, all clinics must have a treatment room. Accommodation is also recommended for venepuncture-local practice will decide whether this is used for all venepunctures. There is space in CE rooms for venepuncture, and some staff prefer this. All clinics in England should have provision for colposcopy which can be in shared or dedicated accommodation.

Unlike other OPDs, staff are permanently based in the medium and large clinics. A pleasant working environment with high quality adequate sized staff rooms and offices will help to recruit, motivate and retain staff by generating an atmosphere which encourages communication and a positive attitude towards the practice of modern high standard GUM. Offices must include space for computers. Many clinics in England have installed computers for patient index, production of $\mathrm{D}$ of $\mathrm{H}$ returns and for other purposes; for reasons of confidentiality these data are best held in the clinic rather than on central computers. Management schemes to implement the proposals in the Government's plans for the health service in England ${ }^{11}$ will require greater computerisation.

There is no specific mention of patients infected with human immunodeficiency virus as they do not require special building facilities for outpatient consultations. Space required will vary according to clinic size and patient numbers. Small clinics with few patients will be able to absorb them into their standard accommodation. Large clinics with large numbers may require additional dedicated accommodation.

The guidance given in the Supplement on GUM clinics should be interpreted flexibly. ${ }^{6}$ Though com- 
missioned by the $\mathrm{D}$ of $\mathrm{H}$ for England it is hoped this guidance will be useful to those planning and designing GUM clinics throughout Britain. Similar accommodation problems exist in other countries, for example in Australia. ${ }^{12} \mathrm{We}$ are unaware of a similar publication in any other country so we hope that this guidance will also help planners, architects, clinicians and others involved in providing premises for genitourinary medicine clinics in other countries.

Correspondence to: Dr R N T Thin, Department of Genitourinary Medicine, St Thomas' Hospital, London SE1 7EH, UK

1 Thin RNT. Workloads in genitourinary medicine clinics in England. Genitourin Med 1989;65:376-81.

2 Chief Medical Officer of the Department of Health. On the State of the Public Health. 1989; London: Her Majesty's Stationery Office: $126 ; 31$.
3 Report of the working group to examine workloads in genitourinary medicine clinics. London: Department of Health, 1988.

4 Special treatment clinic; a design guide. London: Department of Health and Social Security, 1974.

5 Health building Note 12 . Outpatients Departments. Department of Health and Welsh Office, 1989.

6 Health building note 12, Supplement 1 , Genitourinary medicine clinics. London, 1989.

7 Augustine CG. Total outpatient attendances and annual number of clinic sessions in genitourinary medicine, England 197787/88. 1989: Department of Health.

8 Local Government Board. Venereal Diseases Circular, London: His Majesty's Stationery Office 1916.

$9 \mathrm{King} \mathrm{A}$. The first Harrison Lecture. The life and times of Colonel Harrison. Br J Venereal Dis 1974;50:391-403.

10 Committee on Genitourinary Medicine. Future manpower requirements and maintenance of standards of clinical care in departments of genitourinary medicine. London: Royal College of Physicians, 1986.

11 Secretaries of State for Health, Wales, Northern Ireland and Scotland. Working for patients. 1989; London: Her Majesty's Stationery Office.

12 Denham I, Plummer D. Future of the Melbourne STD service. Venereology 1989;2:31.

Accepted for publication 4 June 1990 